set theory, and deal with intersection, complement, power set, etc.

Chapter I of this study includes a statement of the axioms of $\Sigma$ together with a few preliminary notions, such as ordered pair and single-valued class of ordered pairs. Chapter II is concerned with the following topics: extension of propositional functions, definition and properties of relations and functions, and properties of operations with classes and sets. Chapter III is devoted to an exposition of ordinal numbers (based on the work of J. von Neumann) in which an ordinal $\alpha$ is the class of all ordinals less than $\alpha$; for example, $0=$ the null set, $1=\{0\}, 2=\{0,1\}$, etc. Chapter IV develops the theory of cardinal numbers. In Chapters $\mathrm{V}$ and VI a model $\Delta$ is constructed for the system $\Sigma$ on the assumption that $\Sigma$ is consistent, for the classes and sets of $\Delta$ are merely those classes and sets of $\Sigma$ characterized as constructible (the definition of constructibility being based on certain properties of ordinal numbers). Let $L$ denote the class of all constructible sets in the universal class $V$. In Chapter VII it is proved that, when the axioms of $\Sigma$ are applied just to the model $\Delta, V$ being the class of all sets in $\Delta$ and $L$ being the class of sets constructible within $\Delta$, then $V=L$, i.e., every set in $\Delta$ is constructible. Thus, if there exists a model for the system $\Sigma$, then there also exists a model for the system $\Sigma$ augmented by the axiom $V=L$, this latter model consisting of the classes and sets constructible within the given model for $\Sigma$. The existence of this latter model shows that, if the system $\Sigma$ is consistent, then the augmented system $\Sigma^{\prime}$ is consistent, where $\Sigma^{\prime}$ comprises the axioms of $\Sigma$ together with the axiom $V=L$. In the last chapter it is proved that the axiom of choice and Cantor's generalized continuum hypothesis are deducible as theorems in the system $\Sigma^{\prime}$.

While a number of subjects are discussed in this study, the material actually developed is largely confined to just those lemmas needed to attain the final result.

\title{
C. C. Torrance
}

Metric Differential Geometry of Curves and Surfaces. By Ernest Preston Lane. Chicago, University Press, 1940. $216+8$ pp. $\$ 3.00$.

This book is designed as a text for beginning graduate students; it is not intended to be an exhaustive treatise on the subject. The order and content of the material has been used by the author in his classes for several years. It has therefore been thoroughly subjected to the acid tests of the classroom for teachability and for readability. It should on that account be of much service to the classroom teacher and to the student in his independent reading.

Although the material selected is classic, the point of view in pres- 
entation is definitely modern. Definitions are so given as to be valid in projective space or in hyperspace if such definitions are possible. Scattered throughout the text are some 330 exercises. Many of these exercises are mere applications of the material in the text proper, but many require developments found in the textual material in the classic treatises. Several of these exercises show the influence of projective methods and material on the author.

The subject matter is well indicated by the chapter headings: Chapter I, Curves; II, The Moving Trihedron; III, Surfaces; IV, Curves on Surfaces; V, Curvature; VI, Transformations of Surfaces. A short bibliography of the classic treatises on Metric Differential Geometry is appended.

Of especial interest is the treatment of the moving trihedron and its application to curves in one-to-one correspondence. Let a curve $C^{\prime}$ be in one-to-one correspondence with a given curve $C$ so that $P^{\prime}$ on $C^{\prime}$ corresponds to $P$ on $C$. The direction cosines of the tangent line, principal normal, and binormal of $C^{\prime}$ at $P^{\prime}$ referred to the local trihedron of $C$ at $P$, are derived as functions of the local coordinates of $P^{\prime}$, the derivatives of the same, and the curvature and torsion of $C$ at $P$. Applications are made to involutes, evolutes, parallel curves, transformation of Combescure, Bertrand curves. Other applications are left to the student as exercises.

In the chapter on surfaces, a lucid exposition is given of envelopes of one parameter families of surfaces. As often occurs, loci associated with space curves are simple when written in local coordinates. Methods of finding envelopes applicable in this case are given. A fresh treatment is also made of ruled surfaces other than developables.

In the chapter on curves on surfaces, the relation between orthogonality, conjugacy, and harmonic separation is stressed. In this chapter too, the Gauss and Weingarten differential equations and the Gauss-Codazzi integrability conditions are derived.

The discussion of the Gaussian and mean curvatures is well presented in Chapter V, the problems of the chapter excellently supplementing the textual material.

Conformal mapping, applicability, equiareal maps, and parallel surfaces are briefly discussed in the concluding chapter. If time permitted for such a discussion, material in this Chapter VI could no doubt have been unified in a manner similar to that used for curves in one-to-one correspondence by using the methods developed by the author in his paper The moving trihedron, Transactions of this Society, vol. 36 (1934), pp. 696-710. 
Although some teachers may fail to find some of the familiar material, the text is ample for a first course. Moreover the exercises give much of the factual material of the missing topics.

The result (7.12) in exercise 2, p. 32 seems to be incorrect. Problem 17 on page 121 would have been more accurately expressed by speaking of an analytic function of a complex variable. The exercises 4 and 5 on page 140 are incorrect. The University of Chicago Press may well feel proud of this book in every respect. It is my hope that the time taken in its preparation will be repaid by its wide use. Written by a master of oral and written exposition it should receive wide acclaim.

Vernon G. Grove

Lattice Theory. By Garrett Birkhoff. (American Mathematical Society Colloquium Publications, vol. 25.) New York, American Mathematical Society, $1940.6+155$ pp. $\$ 2.50$.

This is the first book on the far-reaching subject of lattices. The author has succeeded in giving a comprehensive, yet not too terse, account of the theory of lattices and its relation to other branches of mathematics. The general plan is to devote six chapters to the abstract theory, and three to applications. Chapter I deals with partially ordered systems, Chapter II with lattices and their general properties; the important modular axiom is assumed in Chapter III, and the axiom of complementation is added in Chapter IV. Distributive lattices and Boolean algebras end the abstract theory in Chapters $\mathrm{V}$ and VI. Thus the author takes the reader through the most important general classes of lattices, by imposing successively restrictive conditions. The last three chapters apply lattice theory to function theory, logic and probability theory.

A partially ordered system is introduced in Chapter I as a set $L$ with a binary relation $\geqq$ on $L$ which is reflexive and transitive and has the property that $x \geqq y, y \geqq x$ implies $x=y$. Most important in connection with general partially ordered systems are the principle of duality, viz., that $L$ together with the converse $\leqq$ of the relation $\geqq$ forms a partially ordered system, and a study of the so-called chain conditions, one or both of which are satisfied in many examples. The ascending chain condition asserts that no infinite sequence $\left(a_{i}\right)$ with $a_{i}<a_{i+1}$ exists; the descending chain condition is dual. Many examples of a partially ordered system are cited, a few of which are the set of all subsets of a set, classes of distinguished subsets of a set, real numbers, integers (relative to the relation of divisibility), partitions, and topologies on a space. 